\title{
A Condition for the Diagonalizability of a Partitioned Matrix
}

\author{
Charles R. Johnson* and Morris Newman
}

Institute for Basic Standards, National Bureau of Standards, Washington, D.C. 20234

(March 5, 1975)

When $U$ and $V$ are diagonalizable matrices the diagonalizability of

$$
\left[\begin{array}{ll}
U & N \\
0 & V
\end{array}\right]
$$

is equivalent to the solvability in $X$ of

$$
U X-X V=N .
$$

A corollary and simple generalization are given.

Key Words: Diagonalizable matrix; partitioned matrix

A square complex matrix $A$ is termed "diagonalizable" if and only if $A$ is similar to a diagonal matrix; that is, if and only if there exists a nonsingular matrix $S$ such that $S^{-1} A S$ is diagonal. Our purpose is to prove the following necessary and sufficient condition for the diagonalizability of a partitioned matrix. We shall denote the class of $n$ by $n$ complex matrices by $M_{n}(C)$.

Theorem 1. Suppose $\mathrm{U}, \mathrm{V} \epsilon \mathrm{M}_{\mathrm{n}}(\mathrm{C})$ are diagonalizable. Then

$$
\left[\begin{array}{ll}
\mathrm{U} & \mathrm{N} \\
0 & \mathrm{~V}
\end{array}\right] \text { is similar to } \quad\left[\begin{array}{ll}
\mathrm{U} & 0 \\
0 & \mathrm{~V}
\end{array}\right]
$$

if and only if there is an $\mathrm{X} \epsilon \mathrm{M}_{\mathrm{n}}(\mathrm{C})$ such that $\mathrm{UX}-\mathrm{XV}=\mathrm{N}$.

Proof: Suppose $S, T \epsilon M_{n}(C)$ are invertible and are such that

$$
S U S^{-1}=D \text { and } T^{-1} V T=E
$$

are diagonal. Then

$$
\left[\begin{array}{ll}
U & N \\
0 & V
\end{array}\right] \text { is similar to }\left[\begin{array}{ll}
U & 0 \\
0 & V
\end{array}\right]
$$


if and only if

$$
\left[\begin{array}{ll}
D & S N T \\
0 & E
\end{array}\right] \text { is similar to }\left[\begin{array}{cc}
D & 0 \\
0 & E
\end{array}\right]
$$

and

$$
U X-X V=N
$$

if and only if

$$
D(S X T)-(S X T) E=S N T .
$$

Thus, it suffices to assume from the outset that $U$ and $V$ are diagonal matrices.

Now suppose, first of all, that

$$
\begin{gathered}
U=\operatorname{diag}\left\{u_{1}, \ldots ., u_{n}\right\}, \\
V=\operatorname{diag}\left\{v_{1}, \ldots ., v_{n}\right\},
\end{gathered}
$$

and that

$$
\left[\begin{array}{ll}
U & N \\
0 & V
\end{array}\right] \text { is similar to }\left[\begin{array}{cc}
U & 0 \\
0 & V
\end{array}\right]
$$

where $N=\left(n_{i j}\right) \epsilon M_{n}(C)$. We will show that if $u_{i}=v_{j}$, then $n_{i j}=0$. It follows that if $X=\left(x_{i j}\right)$ is defined by $x_{i j}=\frac{n_{i j}}{u_{i}-v_{j}}$ for $u_{i} \neq v_{j}$, and $x_{i j}$ arbitrary otherwise, then $U X-X V=N$.

Denote by $E_{i j}$ the $n$ by $n$ matrix all of whose entries are 0 except for a 1 in the $i, j$ position. Then

$$
\left[\begin{array}{ll}
I & t E_{i j} \\
0 & I
\end{array}\right]\left[\begin{array}{ll}
U & N \\
0 & V
\end{array}\right]\left[\begin{array}{cc}
I & -t E_{i j} \\
0 & I
\end{array}\right]=\left[\begin{array}{ll}
U & N+t\left(v_{j}-u_{i}\right) E_{i j} \\
0 & V
\end{array}\right] .
$$

Because of this similarity we may assume without loss of generality that $n_{i j}=0$ whenever $v_{j} \neq u_{i}$. If $N=0$, we are finished. If not, we shall reach a contradiction. Suppose $N \neq 0$. Then via a permutational similarity we may assume that

$$
\left[\begin{array}{ll}
U & N \\
0 & V
\end{array}\right]
$$

is such that

$$
N=\left[\begin{array}{ll}
N_{1} & 0 \\
0 & N_{2}
\end{array}\right]
$$


$u_{i}=v_{j}=u$ for $i \leqslant k, j \leqslant \ell$,

$u_{i} \neq u, v_{j} \neq u$ for $i>k, j>\ell$, and

$N_{1} \neq 0$ is $k$ by $\ell$. It is then clear that

$$
\left[\begin{array}{ll}
U & N \\
0 & V
\end{array}\right]
$$

is permutationally similar to

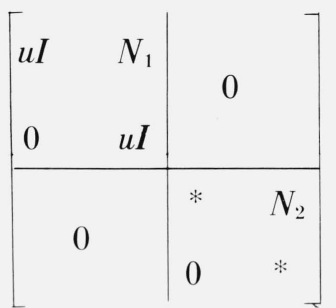

Since

$$
\left[\begin{array}{cc}
u I & N_{1} \\
0 & u I
\end{array}\right] \quad \text { is similar to }\left[\begin{array}{cc}
u I & 0 \\
0 & u I
\end{array}\right]
$$

only if $N_{1}=0$, the assumption that $N \neq 0$ contradicts our original supposition that

$$
\left[\begin{array}{ll}
U & N \\
0 & V
\end{array}\right] \text { is similar to }\left[\begin{array}{cc}
U & 0 \\
0 & V
\end{array}\right] .
$$

Thus our original supposition does imply that $n_{i j}=0$ whenever $v_{j}=u_{i}$ which in turn implies that $U X-X V$ is solvable.

Finally suppose on the other hand that $X \in M_{n}(C)$ is such that

$$
U X-X V=N .
$$

Then it is a simple computation that

$$
\left[\begin{array}{cc}
I & X \\
0 & I
\end{array}\right]\left[\begin{array}{cc}
U & N \\
0 & V
\end{array}\right]\left[\begin{array}{cc}
I & -X \\
0 & I
\end{array}\right]=\left[\begin{array}{cc}
U & -U X+N+X V \\
0 & V
\end{array}\right]=\left[\begin{array}{ll}
U & 0 \\
0 & V
\end{array}\right] ;
$$

and the proof of the theorem is complete.

Corollary. Suppose $\mathrm{U}, \mathrm{V} \epsilon \mathrm{M}_{\mathrm{n}}(\mathrm{C})$ satisfy $\mathrm{U}^{\mathrm{p}}=\mathrm{V}^{\mathrm{p}}=\mathrm{I}, \mathrm{p} \epsilon \mathrm{I}^{+}$. Then

$$
\left[\begin{array}{ll}
\mathrm{U} & \mathrm{N} \\
0 & \mathrm{~V}
\end{array}\right]^{\mathrm{p}}=\mathrm{I}
$$

if and only if there is an $\mathrm{X} \epsilon \mathrm{M}_{\mathrm{n}}(\mathrm{C})$ such that $\mathrm{UX}-\mathrm{XV}=\mathrm{N}$.

Proof: Since $U^{p}=V^{p}=I, U$ and $V$ are diagonalizable and the hypothesis of the theorem is satisfied. Now, if 


$$
\left[\begin{array}{ll}
U & N \\
0 & V
\end{array}\right]^{\prime \prime}=I,
$$

then

$$
\left[\begin{array}{cc}
U & N \\
0 & V
\end{array}\right] \text { is diagonalizable }
$$

and thus similar to

$$
\left[\begin{array}{ll}
U & 0 \\
0 & V
\end{array}\right]
$$

By the theorem, this implies $U X-X V=N$ is solvable. Conversely, if $U X-X V=N$, then

$$
\left[\begin{array}{ll}
U & N \\
0 & V
\end{array}\right]^{p} \quad \text { is similar to } \quad\left[\begin{array}{cc}
U & 0 \\
0 & V
\end{array}\right]^{p}
$$

which is equal to $I$. thus

$$
\left[\begin{array}{ll}
U & N \\
0 & V
\end{array}\right]=I
$$

which completes the proof of the corollary.

A straightforward generalization of Theorem 1 is as follows.

Theorem 2. Suppose each $\mathrm{U}_{\mathrm{i}} \epsilon \mathrm{M}_{\mathrm{n}}(\mathrm{C}), \mathrm{i}=1, \ldots, \mathrm{k}$, is diagonalizable. Then

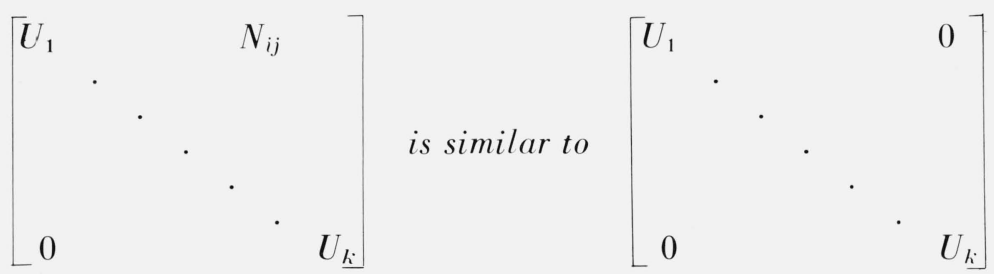

if and only if for each $\mathrm{i}, \mathrm{j} \leqslant \mathrm{k}$ there is an $\mathrm{X} \epsilon \mathrm{M}_{\mathrm{n}}(\mathrm{C})$ (depending on $\mathrm{i}$ and $\mathrm{j}$ ) such that $\mathrm{U}_{\mathrm{i}} \mathrm{X}-\mathrm{XU}_{\mathrm{j}}=\mathrm{N}_{\mathrm{ij}}$.

(Paper 79B1 \& 2-419) 